\title{
Epidemiology of Sporadic Cases of Human Enterovirus 92 in Captivated Primates with Acute Diarrhea
}

\author{
Weikun Zeng ${ }^{1}$, Xianchen Zhao ${ }^{1}$, Wenhai Yu ${ }^{2}$, Zhanlong $\mathrm{He}^{2}$, Shenrong Jing ${ }^{1}$, Fen Huang ${ }^{1, *}$ \\ ${ }^{1}$ Medical Faculty, Kunming University of Science and Technology, Kunming, China \\ 2 Institute of Medical Biology, Chinese Academy of Medical Sciences and Peking Union Medical College, Kunming, China \\ ${ }^{*}$ Corresponding author: Fen Huang, Medical Faculty, Kunming University of Science and Technology, Kunming, China. Tel: +86-87165920776, Fax: +86-87165920776, E-mail: huang- \\ fen6789@163.com.
}

Received: August 20, 2012; Revised: December 22, 2012; Accepted: January 12, 2013

\begin{abstract}
Background: Enteroviruses are among the most common viruses, which cause more than one billion infections worldwide. Enterovirus 92 (EV92) is a newly identified virus, about which few epidemiological data are available.

Objectives: The aim of this study was to investigate the echoviruses in captive rhesus macaques (Macaca mulatta) with acute diarrheal disease in the southwest of China.

Materials and Methods: Stool samples (246) of diarrheal monkeys were collected for detection of EV92 by reverse transcription PCR(RTPCR) as well as analysis of the epidemiological characterizations.

Results: Sequence analysis indicated that echovirus shared the highest nucleotide identity (85\%) with the EV92 strain isolated from the rhesus macaques with gastroenteritis from a US primate center. Phylogenetic analysis showed that the EV92 isolated in this study had clustered together with other echoviruses isolated from the primates.

Conclusions: The results suggested that EV92 as a new echovirus may cause acute diarrhea in M. mulatta.
\end{abstract}

Keywords: Echovirus; Macaca mulatta; Diarrhea

\section{Background}

Members of the Echovirus genus belonging to the Picronaviridae family are among the most common disease-causing viruses in humans, including echoviruses, coxsackieviruses, polioviruses and other echoviruses (1). More than 200 serotypes of echoviruses have been recognized (2). Echoviruses infect several mammals, humans, non-human primates, cattle and pigs (1). Echovirus infection is associated with significant morbidity and mortality in humans, especially in neonates (3). Molecular biology methods based on RT-PCR have recently been developed for echovirus typing (4), which may result in a more conclusive diagnosis compared to the neutralization of virus isolates in cell culture using standardized antisera (5). The neutralization method has been replaced by the sequence of VP1 region as the gold standard of echovirus typing (5). Meanwhile, the typing strategy, based on the partial sequencing of 5' non-coding region (5'-NCR), has been developed to predict the type of echovirus, which is concordant with the VP1 region dependent analysis (6).

Simian echoviruses were originally isolated from primary cell cultures or captivated and wild-caught nonhuman primates in 1950 s and 1960 s $(5,7,8)$. Some simian echoviruses are classified in the species of human echovirus A (HEV-A) on the basis of their sequence relationships to the human echoviruses in HEV-A, such as simian echovirus 46 and human echovirus 92 (7, 9). Human echovirus 92 (EV92), newly typed in 2008, was first detected in diarrheal rhesus macaques and pigtailed macaques (9).EV92 was detected more frequently than other types of echoviruses in simian diarrhea (9). However, there are limited information about the epidemiology of EV92 in the Southwest China, where most of the primates were harbored.

\section{Objectives}

From June 2008, Macaca mulatta, captured from the wildlife of the southwest China were affected by acute diarrhea and/or acute flaccid paralysis. To investigate the causative agents, primer pairs were designed based on the 5'-NCR of EV92 to detect the echovirus using RT-PCR method.

\section{Materials and Methods}

\subsection{Stool Samples}

Fresh stool samples (246) of diarrhoeal M. mulatta were

Implication for health policy/practice/research/medical education:

Human enterovirus 92 (EV92), newly typed in 2008, was first detected in diarrheal rhesus macaques and pigtailed macaques. EV92 was detected more frequently than other types of enteroviruses in diarrheal simian. However, there are limited information about the epidemiology of EV92 in the Southwest China, where most of the primates were harbored.

Copyright (c) 2013, Ahvaz Jundishapur University of Medical Sciences; Published by Kowsar Corp. This is an open-access article distributed under the terms of the Creative Commons Attribution License, which permits unrestricted use, distribution, and reproduction in any medium, provided the original work is properly cited. 
collected for the echovirus detection. Samples were stored at $-70^{\circ} \mathrm{C}$ until used.

\subsection{RT-PCR Detection}

All the samples were suspended in phosphate-buffered saline (PBS), pH 7.4 with $0.01 \%$ diethyl pyrocarbonate (DEPC), at a proportion of $10 \%$ (w/v). Total RNA was extracted from each sample by Trizol (Invitrogen, America) in accordance with the manufacturer's directions. cDNA was transcribed from the total RNA ( $~ 3$ ug) using an AMV Reverse Transcriptase XL for RT-PCR (Takara, Japan) according to the manufacturer's directions. The reverse transcription primer was P2: 5'-CTGTGTGTGTAGAATCTGTTC-3'. RT-PCR was performed using the following primers: P1: 5'-TTTAAAACAGCCTGTGGGTTG-3' and P2. The re- verse transcription protocol was performed at $30^{\circ} \mathrm{C}$ for 10 minutes, $42^{\circ} \mathrm{C}$ for 30 minutes and $99^{\circ} \mathrm{C}$ for 5 minutes. The resulting cDNA $(2 \mu \mathrm{l})$ was amplified by PCR at $94^{\circ} \mathrm{C}$ for 2 minutes, followed by $94^{\circ} \mathrm{C}$ for 30 seconds, $50^{\circ} \mathrm{C}$ for $30 \mathrm{sec}-$ onds and $72^{\circ} \mathrm{C}$ for 1 minute, and the reaction was repeated for 29 cycles. The PCR products were detected by electrophoresis on agarose gel containing $0.5 \mu \mathrm{g} / \mathrm{mL}$ ethidium bromide. The expected DNA band, specific for the EV92, was extracted from the gel, purified with the AxyPrep DNA Gel Extraction kit (Axygen, USA) and cloned into the pMD 18 T-Vector (TaKaRa, Japan). Both strands of each inserted DNA amplicon were sequenced in a DNA analyzer (Applied Biosystems 3730 DNA Analyzer; Invitrogen, USA). Standard precautions were used for all procedures to reduce the possibility of sample contamination and RNA degradation.

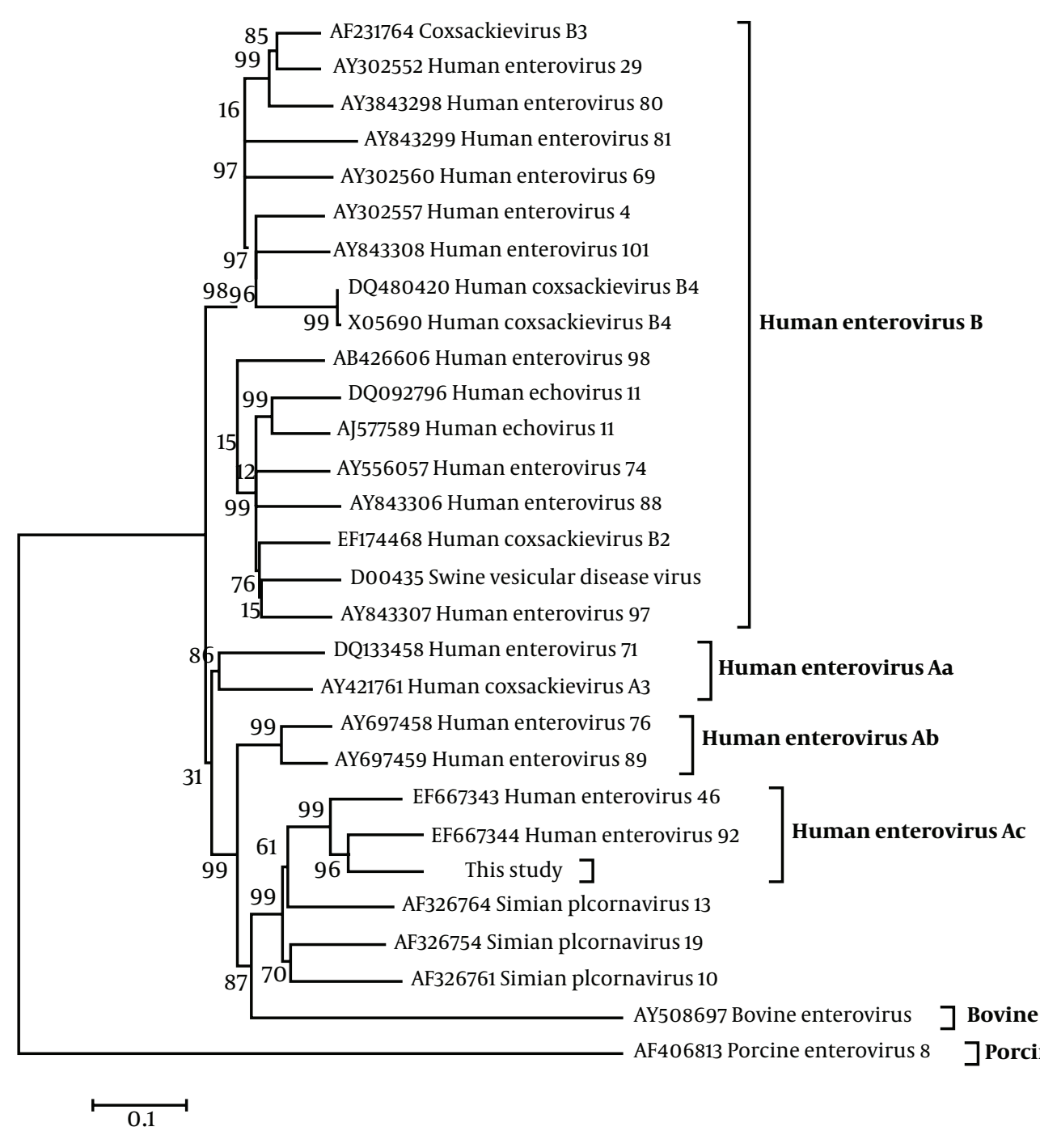

Simian picomavirus

Figure 1. Phylogenetic Analysis Based on the 1105 base pair (bp) Sequence of the Isolates of This Study and Other 28 References From the Genus of Enterovirus, Using the Neighbor-Joining Method. 


\subsection{Sequence Analysis}

Sequences were aligned using Clustal X 1.8 or MegAlign program in the DNASTAR software package. Phylogenetic tree was constructed using the Mega 4 software, (http:// www.megasoftware.net/) using the neighbor-joining method. A maximum-likelihood tree that showed the similar clustering is not shown.

\section{Results}

The sequence analysis suggested that one specimen from a four years old monkey was positive for EV92 RNA, which belongs to the human echovirus A group (HEV-A). The nucleotide sequence contained 1105 nucleotides, including the 5' NCR, VP4 region and partial VP2 region, which shared the highest similarity (85\%) with human echovirus 92 (GenBank No. EF667344.1). The coding region encoded a potential 134 -amino acid poly-protein.

To investigate the evolutionary relationship of the virus isolated from the diarrhoeal monkey, phylogenetic relationships of the sequence were examined using twenty-eight echovirus reference strains (including simian, human, porcine and bovine echovirus original isolates) and the complete genome obtained from GenBank (Figure 1). The prototype of porcine echovirus (GenBank No. AF406813) and bovine echovirus (GenBank No. AY508697) were used as the out-group viruses for construction of the phylogenetic tree. Sequence analysis indicated that the isolate shared high identities with other reported simian echovirus isolates and the highest identity with EV92 (GenBank No. EF667344). Phylogenetic analysis indicated that the EV92 isolated in this study, cluster together with EV92 and SV46 isolates, separated in a US primate center ( 10 ), as shown in Figure 1 Human echovirus Ac subgroup. The tree was evaluated using the interior branch test method with Mega 4 software. Percent bootstrap support is indicated at each node. The scale bar represents the nucleotide substitution per base. GenBank accession No. and name of the virus are indicated. The isolate identified in this study is marked with a triangle

\section{Discussion}

Most echovirus infections are asymptomatic or just cause mild illnesses, but some echovirus es such as some strains of EV76, EV89, EV90, EV91 and EV92, can cause clinical illnesses, including acute diarrhea and acute flaccid paralysis ( 9 - 11). In the present study, EV92 was detected in the stool of primates (rhesus macaques and pigtail macaques) possessing gastroenteritis. EV92 caused acute diarrhea in rhesus macaques, the clinical symptoms of which were similar to the previous studies $(7,9,11)$. The phylogenetic relationships among human EV71 echoviruses (Figure 1) (Human echovirus, Aa subgroup), EV76 and EV89 (Figure 1) (Human echovirus, Ab subgroup), and EV92 isolated in this study (Figure 1) (Human echovirus, Ac subgroup), clustered in a unique phylogenetic cluster- ing, suggested an unexplored relationship between human and simian echovirus es.

Previously, studies suggested that some simian echoviruses, SV19, SV46 and EV92 had little distinction between human and simian echovirus stains, and transmission from non-human primates to humans is possible $(9,11)$. A high prevalence of EV92 (about $56.7 \%$, 17/30) had been reported in diarrhoeal primates in a US primate center $(9,11)$.

In conclusion, although only one EV92 isolate was detected in this study $(0.4 \%, 1 / 246)$, EV92 breakout should be promptly alerted in this region of China. EV92 might be a new echovirus in non-human primates, causing acute diarrhea, and the probability of transmission across species should be warned.

\section{Acknowledgements}

We thank Institute of Medical Biology, Chinese Academy of Medical Sciences and Peking Union Medical College, for their collection of samples.

\section{Authors' Contribution}

Wenhai Yu and Zhanlong He collected samples, Weikun Zeng and Xianchen Zhao performed detection. Fen Huang and Shenrong Jing analyzed the data and wrote the manuscript.

\section{Financial Disclosure}

There is no financial disclosure.

\section{Funding/Support}

This work was supported by National Natural Science Foundation of China (31360619), Natural Science Foundation of Yunnan province in China (2011FZ0s68, 2013FB032 and 2013FZ142) and Foundation of Yunnan Educational Committee (2011Y382).

\section{References}

1. Bruu A-L. Enteroviruses: Polioviruses, Coxsackieviruses, Echoviruses and Newer Enteroviruses. A Practical Guide to Clinical Virology, Second Edition. 2003:44-45.

2. Mayo MA, Pringle CR. Virus taxonomy-1997. J Gen Virol. 1998;79 ( Pt 4):649-57.

3. Tebruegge M, Curtis N. Enterovirus infections in neonates. Semin Fetal Neonatal Med. 2009;14(4):222-7.

4. Oberste MS, Maher K, Kilpatrick DR, Pallansch MA. Molecular evolution of the human enteroviruses: correlation of serotype with VP1 sequence and application to picornavirus classification. J Virol.1999;73(3):1941-8.

5. Oberste MS, Pallansch MA. Enterovirus molecular detection and typing. Rev Med Microbiol. 2005;16:163-171.

6. Richter J, Koptides D, Tryfonos C, Christodoulou C. Molecular typing of enteroviruses associated with viral meningitis in Cyprus, 2000-2002.J Med Microbiol. 2006;55(Pt 8):1035-41.

7. Nix WA, Jiang B, Maher K, Strobert E, Oberste MS. Identification of enteroviruses in naturally infected captive primates. J Clin Microbiol. 2008;46(9):2874-8.

8. Oberste MS, Maher K, Pallansch MA. Molecular phylogeny and proposed classification of the simian picornaviruses. J Virol. 
2002;76(3):1244-51.

9. Oberste MS, Jiang X, Maher K, Nix WA, Jiang B. The complete genome sequences for three simian enteroviruses isolated from captive primates. Arch Virol. 2008;153(11):2117-22.

10. Oberste MS, Maher K, Michele SM, Belliot G, Uddin M, Pallansch MA. Enteroviruses 76, 89, 90 and 91 represent a novel group within the species Human enterovirus A. J Gen Virol. 2005;86(Pt 2):445-51.

11. Oberste MS, Maher K, Pallansch MA. The complete genome sequences fornine simian enteroviruses isolated from captive primates. J Gen Virol. 2007;86(2):3360-72. 